\title{
Evaluación de impacto de los recursos informativos bibliotecarios en la competitividad académica mediante ecuaciones estructurales
}

\author{
Assesing the academic competitiveness impact of library information resources with structural equation models
}

\author{
José Refugio Romo-González, Laura Patricia MuRguía JÁQUeZ, \\ Javier TARANGO y Juan D. MACHIN-MASTROMATTEO
}

Universidad Autónoma de Chihuahua, Facultad de Filosofía y Letras, Campus Universitario \# 1, Chihuahua, Chih., México, C.P.31130, Apartado Postal 744, jromo@uach.mx, pmurguia@uach.mx, tj.88888@hotmail.com, jmachin@uach.mx

\begin{abstract}
Resumen
Se evalúan de manera exploratoria y predictiva las relaciones causales y el impacto que tienen los distintos recursos informativos bibliotecarios (impresos, digitales, servicios y espacios físicos) en la competitividad académica (productividad, calidad e innovación) de 33 universidades mexicanas acreditadas, aplicando modelos de ecuaciones estructurales (SEM) con la técnica de cuadrados mínimos parciales (PLS). Los resultados muestran que algunos de los recursos informativos impactan positivamente a la productividad y la calidad académica, más no así a la innovación. El análisis multivariado PLSSEM demostró ser muy valioso para evaluar las relaciones causales en los modelos propuestos, por lo que se recomienda ampliamente su empleo.

Palabras clave: Bibliotecas universitarias. Recursos informativos. Competitividad académica. Evaluación del impacto. PLS-SEM. México.
\end{abstract}

\section{Introducción}

Existe una preocupación antaña por conocer y describir la situación de las bibliotecas académicas, particularmente las universitarias. Más recientemente las indagaciones se dirigieron hacia sus indicadores de eficacia y eficiencia, pero actualmente la preocupación fundamental tiende hacia las evaluaciones de impacto. En México, la Secretaría de Educación Pública (SEP) y las propias universidades públicas del país, han mantenido un interés constante por dotar de mayores recursos y ofrecer mejores servicios bibliotecarios para sus comunidades. Sin embargo es necesario, ante la ingente escasez de recursos y la transparencia requerida en su uso, evaluar el impacto real de estos recursos y servicios sobre el desempeño institucional, particularmente sobre la competitividad universitaria, que según Palacios y Vargas (2009, p. 19) se integra y sustenta por tres elementos que son condiciones para lograrla: productividad, calidad e innovación.

\begin{abstract}
The causal relationships and impact of the different librarian information resources (printed, digital, services and physical spaces) on the academic competitiveness (productivity, quality and innovation) of 33 accredited Mexican universities are evaluated applying structural equation models (SEM) with the technique of partial least squares (PLS). The results show that some of the information resources positively impact productivity and academic quality, but not innovation. The PLSSEM multivariate analysis proved to be very valuable in assessing the causal relationships in the proposed models, so its use is widely recommended.
\end{abstract}

Keywords: University libraries. Impact assessment. Information resources. Academic competitiveness. PLS-SEM. Mexico.

Este estudio tiene como objetivo principal evaluar de manera exploratoria y predictiva el impacto de los recursos informativos bibliotecarios sobre la competitividad de universidades acreditadas en México, tanto públicas como privadas, a través de una modelación con ecuaciones estructurales y cuadrados mínimos parciales, que es un método estadístico multivariante que analiza las causas y efectos de un fenómeno dado, procedente de una investigación no experimental. La información referente a los recursos informativos de las bibliotecas es de aquellas universidades acreditadas ante el Consejo Nacional para Asuntos Bibliotecarios de Instituciones de Educación Superior (CONPAB-IES, 2015) y los datos sobre la competitividad académica (productividad, calidad e innovación) provienen del Estudio Comparativo de Universidades Mexicanas (ECUM) desarrollado por la Universidad Nacional Autónoma de México (UNAM, 2016), seleccionando los indicadores respectivos de acuerdo a su consistencia y homogeneidad. 


\section{Revisión de literatura}

El estudio de la influencia de las bibliotecas académicas y particularmente de las universitarias, en el desarrollo de las actividades sustantivas de dichas instituciones, ha sido abordado previamente por diversos autores (Lau, 2001; Tarango y Hernández, 2009), destacando por ser una tarea compleja, no sólo por la recolección de los datos mismos, sino también por los métodos empleados. La contribución de las bibliotecas universitarias, en el caso de la actividad institucional de investigación científica, ha dado pie incluso a sentar las bases para el desarrollo de un modelo de evaluación de este tipo de bibliotecas (Mireles et. al., 2009) y desde luego al estudio de la influencia de la infraestructura bibliotecaria en la producción científica de universidades y centros de investigación (Murguía et. al., 2013), encontrándose correlacionados. Fernández y Rubio (2013), preocupados por el presupuesto asignado a las bibliotecas, estudian su relación con la productividad investigadora de la universidad, no encontrando correlación significativa entre dichas variables de una manera general, pero si encuentran una fuerte asociación entre el presupuesto para recursos en formato electrónico y la productividad investigadora.

La eficiencia y efectividad de los servicios de información no sólo tienen que ver con sus recursos electrónicos, sino también con la propia arquitectura bibliotecaria (Mireles y Arellano, 2015), por lo que es necesario establecer y seguir cierta normatividad en la construcción y adaptación de los edificios que los albergan para que sus espacios sean seguros y funcionales, e incluso estéticos con la finalidad de promover el rendimiento académico, la creatividad y la interacción de las distintas comunidades universitarias. Por otro lado, las nuevas tecnologías de la información y la comunicación (TIC), están promoviendo cambios sustanciales en los servicios que deben prestar las bibliotecas (Tovar-Sanz, 2015) e incluso cambios en su infraestructura física, por lo que se debe seguir invirtiendo en la capacitación del personal, sino se quiere quedar en el rezago. Finalmente, está ocurriendo un llamado a que las bibliotecas universitarias orienten sus modelos de servicios informativos bajo una perspectiva de mercadotecnia (González-Solar, 2016), es decir que se adapten centralmente en las necesidades y requerimientos de los usuarios, pues de otra manera sus servicios dejarán de ser percibidos como un factor importante de la competitividad de las universidades.

\section{Planteamiento del problema}

La evaluación del impacto de los recursos informativos bibliotecarios sobre las actividades sustantivas de las universidades es algo no sólo necesario sino imprescindible hoy en día, pero para ello es necesario contar con métodos, técnicas y herramientas confiables y válidas, que justamente midan el fenómeno desde una óptica causa - efecto, superando la limitada visión diagnóstica - descriptiva, frecuentemente empleada pero muy corta en sus alcances. Por lo tanto, la pregunta principal de investigación de este trabajo se concentra en saber si la modelación con ecuaciones estructurales (SEM) bajo la técnica de cuadrados mínimos parciales (PLS), puede establecer a partir de un modelo teórico conceptual cuál es el impacto real y causal de los recursos informativos bibliotecarios sobre la competitividad universitaria.

\subsection{Justificación}

Ante los fenómenos de globalización y rendición de cuentas en la nueva sociedad de la información y el conocimiento, las universidades como instituciones encargadas de la generación, transmisión y transferencia del conocimiento, dirigen sus esfuerzos hacia el logro de niveles cada vez más altos en su competitividad, conformada por tres pilares básicos: productividad, calidad e innovación. Las bibliotecas o centros de información de las universidades, como un punto nodal de apoyo a sus principales actividades de docencia, investigación y difusión de la cultura, deben participar activamente y de una manera armónica en el cumplimiento de su misión y alcance de su visión, para lo cual deben optimizar el uso de los recursos informativos bibliotecarios, impactando de una manera fehaciente en el desempeño de los profesores - investigadores y obviamente en el rendimiento de sus estudiantes. Por lo anterior, es necesario contar con estudios que demuestren en primera instancia el impacto de los recursos informativos bibliotecarios en la competitividad académica universitaria, con fines de lograr una mayor y mejor asignación presupuestal, pero también para realizar un manejo más racional y eficiente de dichos recursos.

\subsection{Objetivos}

Se plantean tres objetivos: evaluar en una primera fase exploratoria y predictiva, el impacto de los recursos informativos bibliotecarios en la competitividad académica de universidades mexicanas acreditadas ante CONPAB-IES; analizar la competitividad académica bajo tres criterios básicos — productividad, calidad e innovación-; y evaluar la significancia y magnitud de los efectos causales de los recursos informativos bibliotecarios (impresos, digitales, servicios e infraestructura) sobre la productividad, calidad e innovación universitaria y por ende sobre su competitividad. 


\subsection{Delimitación}

Para el desarrollo de la investigación fueron seleccionadas 33 universidades mexicanas afiliadas al CONPAB-IES, que es una organización que promueve el desarrollo de los servicios bibliotecarios en las instituciones académicas y de investigación en México, a través de proyectos de colaboración y que a su vez contaran para el mismo período de estudio con datos consistentes sobre su competitividad académica en el ya citado ECUM desarrollado por la UNAM.

\section{Metodología}

El enfoque del trabajo es cuantitivista y de naturaleza no experimental, bajo un diseño de investigación transversal - causal, con un corte anual (2012), empleando un censo de 33 universidades mexicanas, es decir no se trata de una muestra representativa a nivel nacional, tanto públicas como privadas cuyo principal criterio de inclusión es estar acreditadas ante el CONPAB-IES con información consistente sobre sus recursos informativos bibliotecarios impresos, digitales, de servicios e infraestructura e información sobre su competitividad académica procedente del ECUM, midiéndose los dos constructos principales (competitividad y recursos informativos) con los indicadores apropiados y previamente seleccionados.

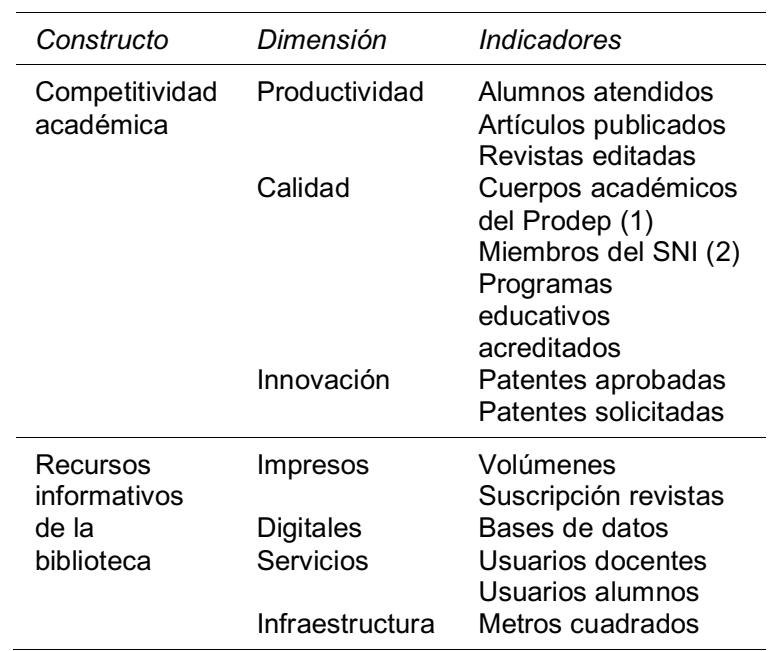

Tabla I. Constructos e indicadores utilizados

La información recabada se sujeta a un análisis de modelación estructural con la técnica de cuadrados mínimos parciales (PLS-SEM) para explorar y predecir las relaciones causales planteadas en un modelo conceptual simplificado y adaptado de Murguía, et. al. (2013, p. 342), usando para ello el programa SmartPLS versión 2.0, http://www.smartpls.de, recomendado en este caso por Lowry y Gaskin (2014, p. 134). La Tabla I muestra los principales constructos e indicadores bajo estudio.

\section{Resultados y discusión}

Los resultados de este trabajo se focalizaron hacia la evaluación del modelo de ecuaciones estructurales que representa el impacto de los recursos informativos bibliotecarios sobre la competitividad académica, por lo que sólo se reportan como resultados descriptivos la distribución de la matrícula escolar entre las 33 universidades estudiadas ( $\mathrm{Fi}$ gura 1, página siguiente), donde se aprecia un rango muy amplio en la matrícula, que va desde los 100 hasta los 90,000 estudiantes atendidos por universidad, así como los índices de competitividad académica por institución (Figura 2, página siguiente), integrados por los respectivos índices de productividad, calidad e innovación ya ponderados y estandarizados. En este caso, las instituciones con los niveles más altos de competitividad fueron El Colegio de México, El Colegio de Michoacán y El Colegio de Sonora, aunque el índice de innovación fue superior en las universidades de Guanajuato, Yucatán, Nuevo León y Colima. La mayoría de las universidades se mantuvo por debajo de los 50 puntos de competitividad académica, cuando el máximo apuntado por El Colegio de México fue de 184 puntos.

Posterior a los resultados descriptivos, se ejecutaron análisis de correlación entre los indicadores de los recursos informativos y los indicadores de competitividad académica. La Figura 3 en la página siguiente ejemplifica el diagrama de dispersión entre el recurso informativo de suscripciones a bases de datos y la competitividad académica, donde la asociación fue altamente significativa $(S i g=0.000)$ y con un nivel de correlación alto $(R=0.690)$. Con respecto a los resultados PLSSEM (Figura 4, página siguiente), se concentraron en los siguientes cinco apartados, según la recomendación de Wong (2013, p. 17) para los modelos con indicadores formativos, además de que en este caso se trata de un modelo de segundo orden.

\subsection{Varianza explicada de la variable endógena}

La variable endógena objetivo en este estudio fue la competitividad académica, la cual es afectada por tres variables latentes exógenas (productividad, calidad e innovación), resultando un coeficiente de determinación $\left(R^{2}\right)$ de 0.973 , lo cual significa que la productividad, calidad e innovación explican sustancialmente el $97.3 \%$ de la varianza de la competitividad. En PLS-SEM, un $\mathrm{R}^{2}$ de 0.75 es sustancial, 0.50 es moderado y 0.25 es pobre. 


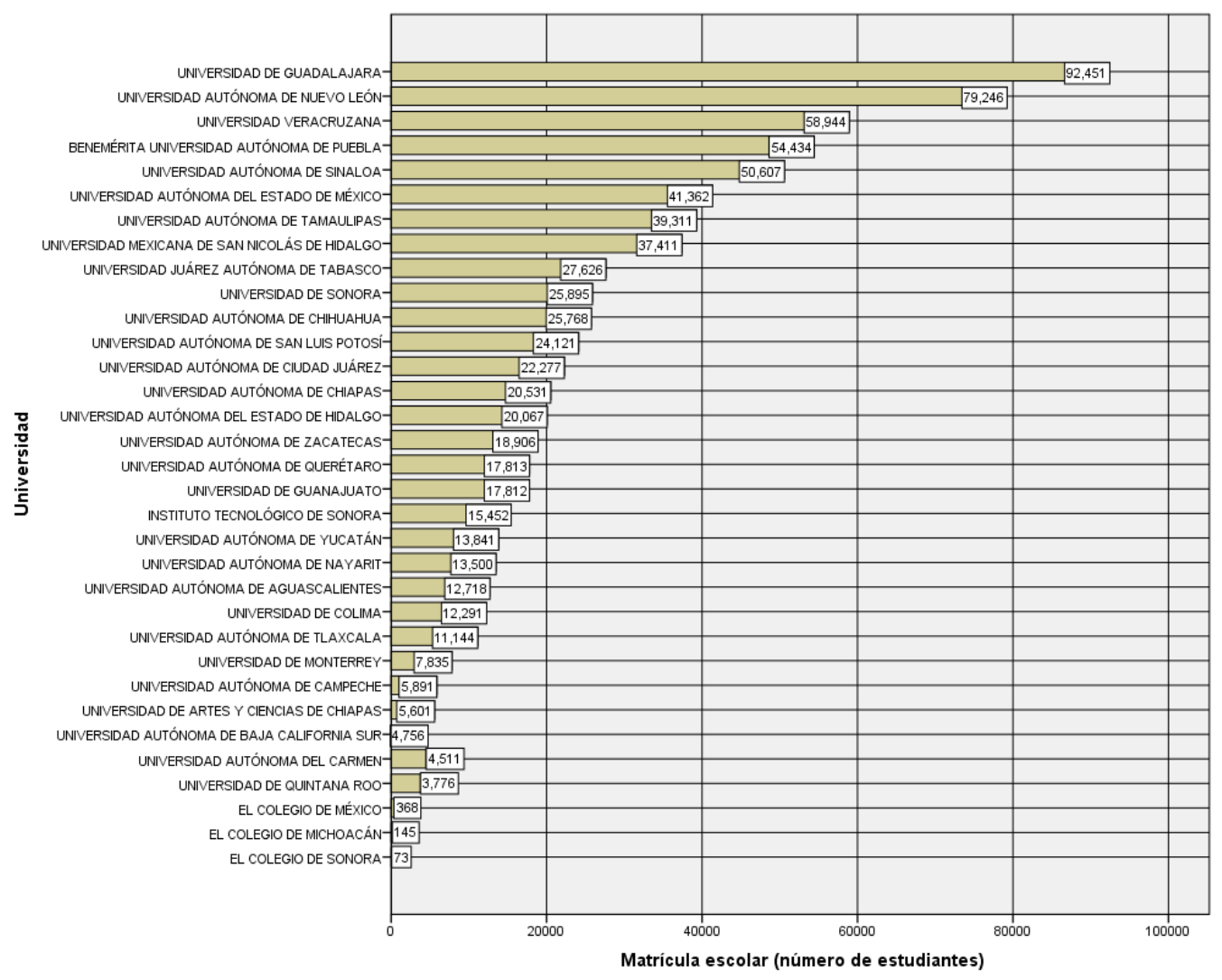

Figura 1. Distribución de matrícula por universidades bajo estudio

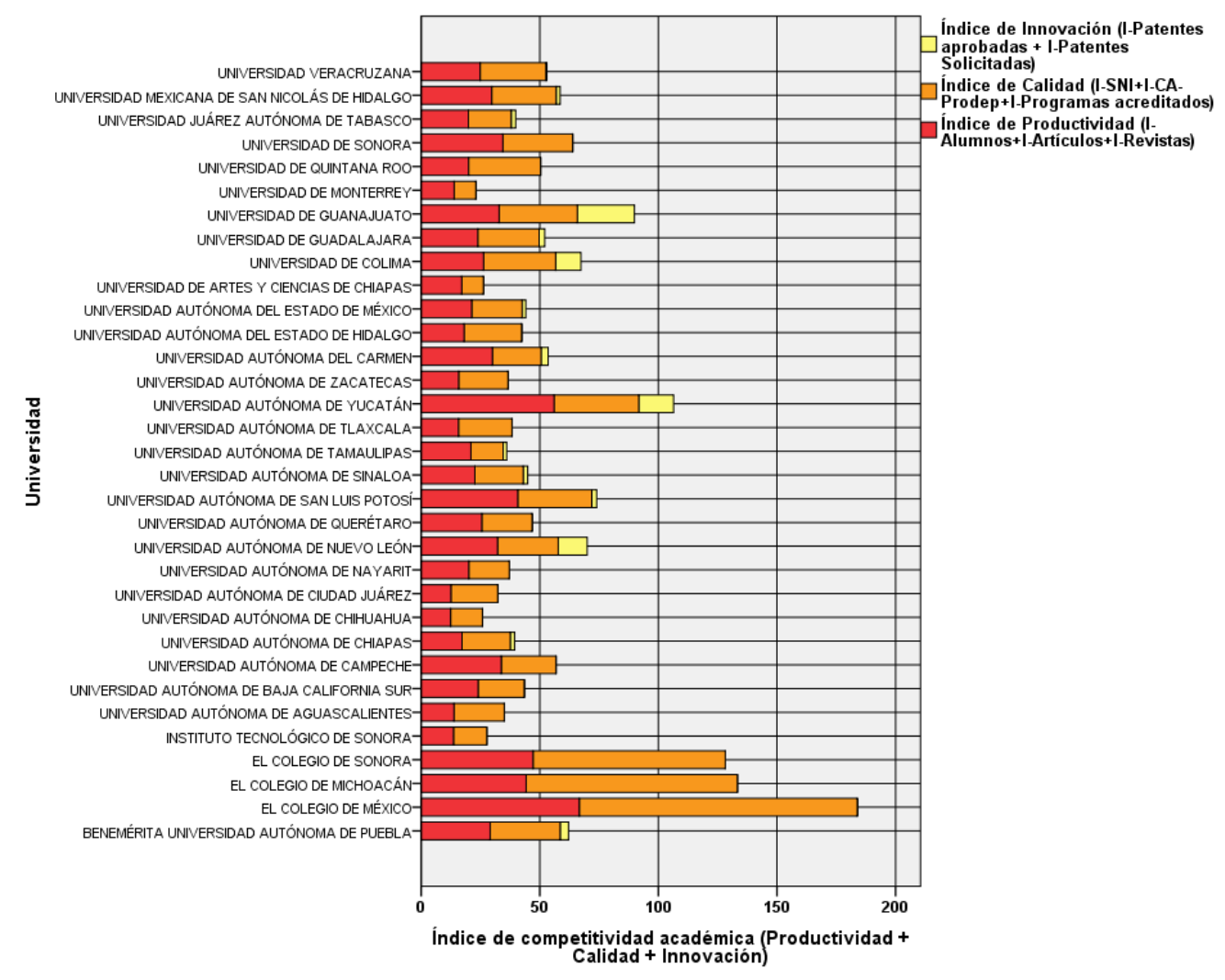

Figura 2. Competitividad académica (productividad, calidad e innovación) por universidades bajo estudio

Romo-González, J. R., Murguía Jáquez, L. P., Tarango, J.; Machin-M, J. D. Evaluación de impacto de los recursos informativos bibliotecarios en la competitividad académica mediante ecuaciones estructurales. // Ibersid. 12:1 (2018) 43-49. ISSN 1888-0967. 


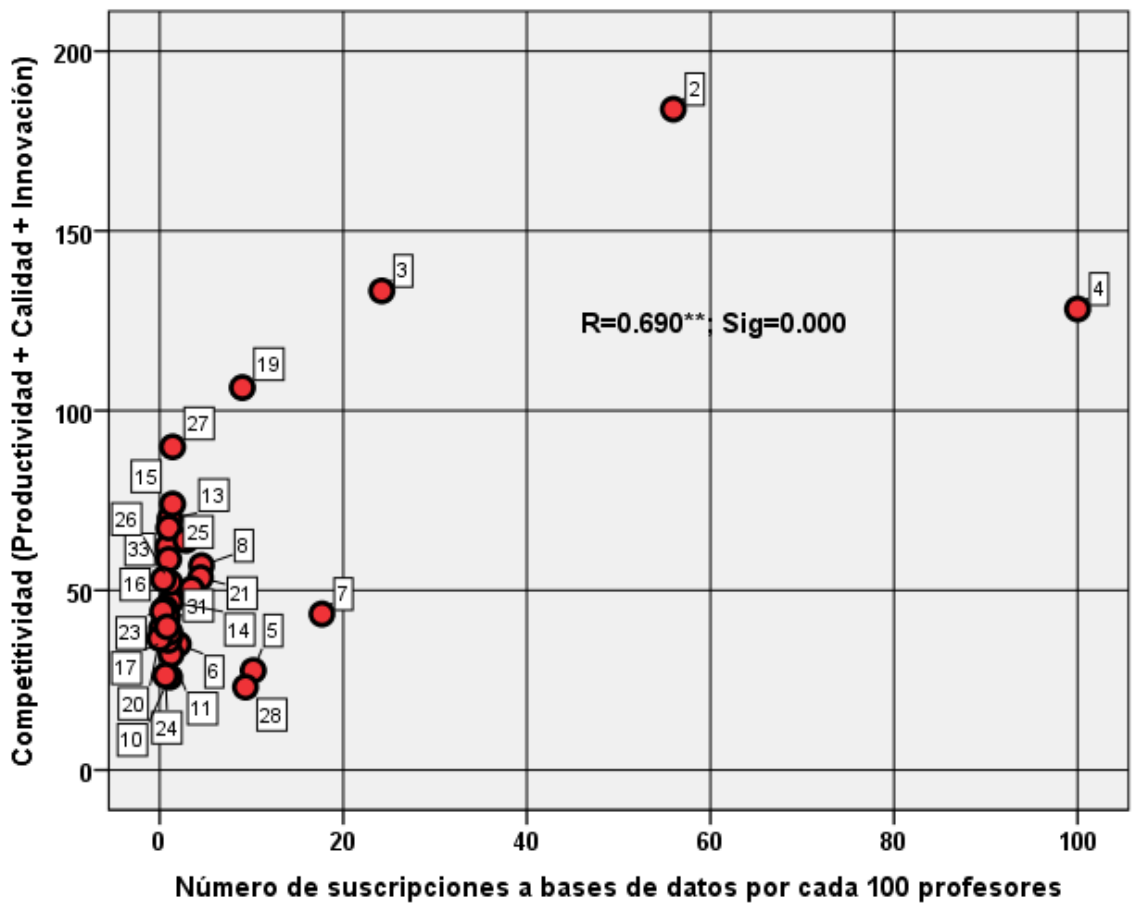

Figura 3. Correlación entre recursos informativos y competitividad académica

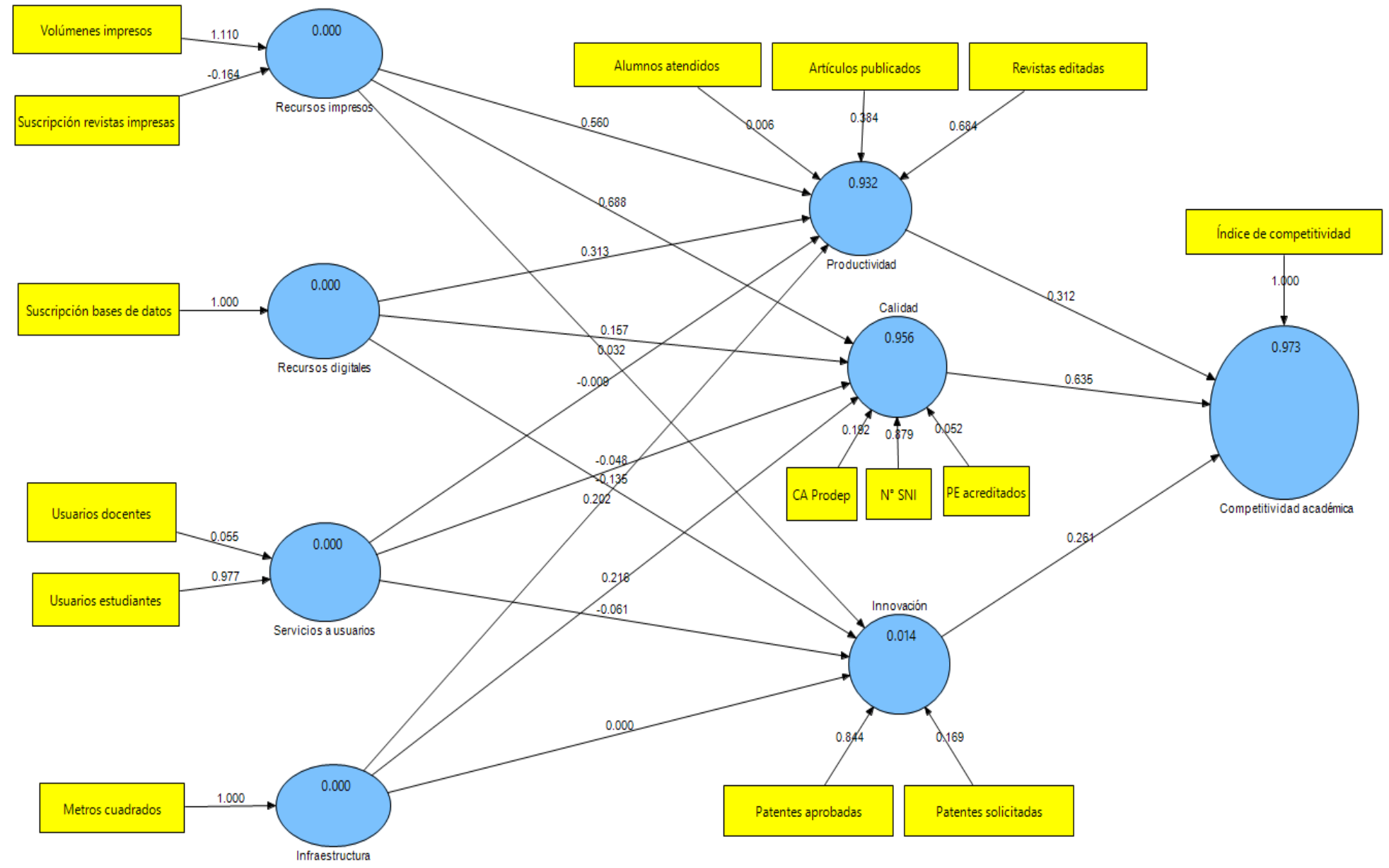

Figura 4. Resultados PLS-SEM: recursos informativos vs competitividad académica 
En el segundo nivel del modelo, la productividad, calidad e innovación son ahora variables endógenas explicadas con las cuatro exógenas que corresponden a los recursos informativos (impresos, digitales, servicios e infraestructura), arrojando explicaciones sustanciales para la productividad (0.932) y la calidad (0.956), pero una explicación prácticamente nula para la innovación (0.014). Es decir, el efecto de los recursos informativos bibliotecarios sobre la innovación universitaria es casi nulo, pero no así sobre su productividad y calidad, donde sus efectos son sustanciales.

\subsection{Magnitud y significancia de las trayectorias estructurales del modelo interno}

En el primer nivel del modelo interno, se puede concluir que los tres predictores de la competitividad son estadísticamente significativos al apuntar coeficientes de trayectoria estandarizados superiores a 0.10 que es el mínimo requerido para la significancia. La calidad es el predictor moderadamente más fuerte (0.636), seguido por la productividad $(0.312)$ y al final por la innovación (0.261).

En el segundo nivel del modelo, los predictores de recursos informativos con efectos significativos y moderadamente fuertes fueron los recursos impresos sobre la calidad (0.688) y productividad (0.560); los recursos digitales con efectos moderados sobre la productividad $(0.313)$ y pobres sobre la calidad (0.157); la infraestructura bibliotecaria tiene un efecto pobre, pero todavía significativo, sobre la calidad $(0.218)$ y la productividad (0.202); los servicios bibliotecarios a usuarios (docentes y estudiantes) no mostraron ningún efecto significativo sobre la calidad y productividad de las universidades y ningún recurso informativo tuvo efectos significativos sobre la innovación.

\subsection{Pesos y significancia del modelo externo}

Las correlaciones entre las variables latentes y sus indicadores en el modelo externo, arrojan los indicadores con mayor peso para explicar la competitividad académica, los cuales pueden verse por orden de importancia en la Tabla II, donde se aprecia que el número de miembros en el SNI, patentes aprobadas, revistas editadas, artículos publicados y cuerpos académicos Prodep son los cinco factores más importantes para elevar la competitividad universitaria y por el contrario, el número de alumnos atendidos, así como el número de programas educativos acreditados son los factores que tienen el impacto más débil en dicha competitividad.

\begin{tabular}{lll}
\hline$N^{\circ}$ & Indicador & Peso \\
\hline $1^{\circ}$ & Número de miembros en el SNI & 0.8792 \\
$2^{\circ}$ & Patentes aprobadas & 0.8444 \\
$3^{\circ}$ & Revistas editadas & 0.6842 \\
$4^{\circ}$ & Artículos publicados & 0.3845 \\
$5^{\circ}$ & Cuerpos académicos Prodep & 0.1921 \\
$6^{\circ}$ & Patentes solicitadas & 0.1691 \\
$7^{\circ}$ & Programas educativos acreditados & 0.0516 \\
$8^{\circ}$ & Número de alumnos atendidos & 0.0059 \\
\hline
\end{tabular}

Tabla II. Indicadores de la competitividad académica

Por otro lado, el algoritmo PLS-SEM logró el criterio de convergencia después de 13 iteraciones (en lugar de las 300 tolerables como máximo), por lo que se considera que la estimación del modelo es buena.

\subsection{Validez de convergencia}

Para verificar este criterio en cada una de las variables latentes, se calculó su varianza extraída promedio (AVE), cuyos valores resultaron mayores a 0.50 , por lo que se confirmó la validez de convergencia en los dos modelos de medición estudiados (recursos informativos y competitividad).

\subsection{Colinealidad entre indicadores}

Como los indicadores empleados en este análisis son de carácter formativo, debe verificarse que no se presentan problemas de colinealidad entre ellos, mostrando la Tabla III los valores del factor de inflación de la varianza (VIF) y su tolerancia para cada indicador de los recursos de información bibliotecarios, donde se puede concluir que no hay problemas de colinealidad pues todos los indicadores apuntan un VIF menor a 5.0 y una tolerancia mayor a 0.20 según los criterios aceptables (Hair, et. al., 2016).

\begin{tabular}{lll}
\hline Indicador & VIF & Tolerancia \\
\hline Recursos impresos & 3.125 & 0.320 \\
Recursos digitales & 2.372 & 0.422 \\
Servicios a usuarios & 1.035 & 0.967 \\
Infraestructura & 1.749 & 0.572 \\
\hline
\end{tabular}

Tabla III. Estadísticos de colinealidad

\section{Conclusiones y recomendaciones}

En general, los resultados mostraron que algunos de los recursos informativos impactan positivamente a la competitividad académica de las universidades mexicanas acreditadas ante el CONPAB-IES, particularmente en su productividad y calidad, más no así en su innovación. 
Específicamente, los recursos informativos con efectos significativos y moderadamente fuertes fueron los recursos impresos (volúmenes y revistas) sobre la calidad (0.688) y la productividad (0.560); los recursos digitales (bases de datos) con efectos moderados sobre la productividad $(0.313)$ y débiles sobre la calidad (0.157); la infraestructura bibliotecaria tiene un efecto pobre, pero todavía significativo, sobre la calidad (0.212) y la productividad (0.202); los servicios bibliotecarios a usuarios (docentes y estudiantes) no mostraron ningún efecto significativo sobre la calidad $(0.048)$ y productividad (0.009) de las universidades y ningún recurso informativo tuvo efectos significativos sobre la innovación $(<0.061)$.

Los indicadores con mayor peso para explicar la competitividad académica, fueron el número de miembros en el SNI (0.879), patentes aprobadas (0.844), revistas editadas (0.684), artículos publicados (0.384), cuerpos académicos Prodep $(0.192)$ y patentes solicitadas $(0.169)$; por el contrario, los factores que tienen el impacto más débil en dicha competitividad son el número de alumnos atendidos (0.005), así como el número de programas educativos acreditados (0.051).

El análisis multivariado PLS-SEM demostró ser muy valioso para evaluar en su fase exploratoria y predictiva las relaciones causales en los modelos propuestos y su ejecución en el programa SmartPLS versión 2.0 resultó ser efectiva y fácil de llevar a cabo, por lo que se recomienda ampliamente su empleo en el campo de la Bibliotecología y Ciencias de la Información.

\section{Notas}

(1) Prodep: Programa para el Desarrollo Profesional Docente para el Tipo Superior de la Secretaría de Educación Pública (SEP). México.

(2) SNI: Sistema Nacional de Investigadores del Consejo Nacional de Ciencia y Tecnología (CONACYT). México

\section{Referencias}

Consejo Nacional para Asuntos Bibliotecarios de Instituciones de Educación Superior, CONPAB-IES. (2015). Diagnóstico de las bibliotecas de instituciones de educación superior e investigación afiliadas al CONPAB-IES 19932013. - La Paz, Baja California Sur: CONPAB-IES, Universidad Autónoma de Baja California Sur. http://www.conpab.org.mx/Documentos/Publicaciones/DiagnosticoBibliotecas\%20CONPAB 2015.pdf

Fernández, Sergio; Rubio, Francisco (2013). ¿El dinero importa? Relación entre el presupuesto de la biblioteca y la productividad investigadora de la Universidad Politécnica de Valencia. // Revista Española de Documentación Científica. 36:4 (Octubre-diciembre 2013, e023) 1-11. http://redc.revistas.csic.es/index.php/redc/article/viewFile/820/1019
Hair, J.F; Hult, G.T; Ringle, C.M.; Sarstedt, M. (2016). A primer on partial least squares structural equation modeling (PLS-SEM). Second edition. Thousand Oaks: Sage.

González-Solar, Llarina (2016) La biblioteca universitaria orientada a la investigación: propuesta de un modelo de servicio centrado en el usuario desde la perspectiva del marketing. Universidad de la Coruña: La Coruña. Tesis Doctoral. http://eprints.rclis.org/30313/1/GonzalezSolar_Llarina_TD_2016.pdf

Lau, Jesús. (2001). Aprendizaje y calidad educativa: el papel de las bibliotecas. Ponencia presentada en Seminario de Bibliotecas y Calidad de la Educación. Medellín: Universidad de Antioquia, May.02, 2001. https://www.researchgate.net/publication/228545060_Aprendizaje_y_calidad_educativa_papel_de_la_biblioteca

Lowry, P. B.; Gaskin, J. (2014). Partial least squares (PLS) structural equation modeling (SEM) for building and test behavioral causal theory: when to choose it and how to use it. // IEEE Transactions on Professional Communication 57:2 (June 2014) 123-146. https://pdfs.semanticscholar.org/6ca7/26d06966a25cc837bfd5fded7f663b9 e9f4e.pdf

Mireles Cárdenas, Celia; Russell Barnard, Jane Margaret; Figueroa Barragán, Laura (2009). Contribución de las bibliotecas universitarias a la investigación científica institucional: bases para el desarrollo de un modelo de evaluación. // Ibersid 3 (2009) 49-53. http://www.ibersid.eu/ojs/index.php/ibersid/article/view/3721/3482

Mireles Cárdenas, Celia; Arellano Rodríguez, J. Alberto (2015). Normatividad sobre arquitectura bibliotecaria en México, elemento imprescindible para la oferta de servicios de información. // Investigación Bibliotecológica 29:66 (Mayo-agosto 2015) 169-196. http://www.revistas.unam.mx/index.php/ibi/article/view/54423/48415

Murguía, Laura; Tarango, Javier; Romo-González, José; Ascencio, Gerardo (2013). Influencia de la infraestructura bibliotecaria en el desarrollo de producción científica en universidades mexicanas inmersas en procesos de acreditación. // Revista General de Información y Documentación. 23:2 (2013) 333-367. http://revistas.ucm.es/index.php/RGID/article/viewFile/43134/40917

Palacios Blanco, José L.; Vargas Chanes, Delfino (2009). Medición efectiva de la calidad, innovaciones en México. México, D.F: Trillas.

Tarango, Javier; Hernández-Orozco, Guillermo. (2009). Evaluación de bibliotecas universitarias: un modelo de avance y desarrollo. // Revista Interamericana de Bibliotecología, 32:2 (Julio-diciembre 2009) 147-175. http://www.scielo.org.co/pdf/rib/v32n2/v32n2a05.pdf

Tovar-Sanz, María (2015). El apoyo a la investigación en las bibliotecas universitarias españolas. // Documentación de las Ciencias de la Información 38 (2015) 311-326. http://revistas.ucm.es/index.php/DCIN/article/view/50822/47169

Universidad Nacional Autónoma de México, Dirección General de Evaluación Institucional (2016). Estudio comparativo de las universidades mexicanas (ECUM). México, D.F: UNAM. http://www.execum.unam.mx/ (2017-01-23).

Wong, K. K. (2013). Partial least squares structural equation modeling (PLS-SEM) techniques using SmartPLS. // Marketing Bulletin. 24, 1-32. http://marketing-bulletin.massey.ac.nz/V24/MB_V24_T1_Wong.pdf

Enviado: 2017-04-01. Segunda versión: 2017-09-23. Aceptado: 2017-10-09. 
